Research article

Open Access

\title{
Increased susceptibility to collagen-induced arthritis in female mice carrying congenic Cia40/Pregq2 fragments
}

\author{
Maria Liljander ${ }^{1}$, Åsa Andersson², Rikard Holmdahl3,4 and Ragnar Mattsson ${ }^{1}$ \\ ${ }^{1}$ Lund Transgenic Core Facility, BMC C13, Lund University, Klinikgatan 28, SE-221 84 Lund, Sweden \\ 2Department of Pharmacology and Pharmacotherapy, Group of Molecular Immunopharmacology, Faculty of Pharmaceutical Sciences, Copenhagen \\ University, Universitetsparken 2, DK-2100 Copenhagen Ø, Denmark \\ ${ }^{3}$ Medical Inflammation Research, Lund University, BMC I11, SE-221 84 Lund, Sweden \\ ${ }^{4}$ Karolinska Institute, Division of Medical Inflammation Research, Sheeles väg 2, SE-171 77 Stockholm, Sweden
}

Corresponding author: Maria Liljander, maria.liljander@med.lu.se

Received: 20 Feb 2008 Revisions requested: 1 Apr 2008 Revisions received: 24 Jun 2008 Accepted: 6 Aug 2008 Published: 6 Aug 2008

Arthritis Research \& Therapy 2008, 10:R88 (doi:10.1186/ar2470)

This article is online at: http://arthritis-research.com/content/10/4/R88

(C) 2008 Liljander et al.; licensee BioMed Central Ltd.

This is an open access article distributed under the terms of the Creative Commons Attribution License (http://creativecommons.org/licenses/by/2.0), which permits unrestricted use, distribution, and reproduction in any medium, provided the original work is properly cited.

\begin{abstract}
Introduction Collagen-induced arthritis (CIA) in mice is a commonly used experimental model for rheumatoid arthritis (RA). We have previously identified a significant quantitative trait locus denoted Cia40 on chromosome 11 that affects $\mathrm{CIA}$ in older female mice. This locus colocalizes with another locus, denoted Pregq2, known to affect reproductive success. The present study was performed to evaluate the role of the Cia40 locus in congenic B10.Q mice and to identify possible polymorphic candidate genes, which may also be relevant in the context of RA.

Methods Congenic B10.Q mice carrying an NFR/N fragment surrounding the Cia40/Pregq2 loci were created by 10 generations of backcrossing (N10). The congenic mice were investigated in the CIA model, and the incidence and severity of arthritis as well as the serum levels of anti-collagen II (CII) antibodies were recorded.

Results Significant effects on onset, incidence, severity, and anti-Cll antibody titers were observed in female mice carrying a heterozygous congenic Cia40/Pregq2 fragment of NFR/N origin, containing one or more polymorphic genes. Congenic male mice did not show increased incidence of CIA, but males carrying a heterozygous fragment showed a significant increase in severity in comparison with wildtype B10.Q males (littermates).

Conclusion The Cia40/Pregq2 locus at chromosome 11 contains one or more polymorphic genes of NFR/N origin that significantly influence both incidence and severity of CIA in heterozygous congenic mice of the B10.Q strain. The major polymorphic candidate genes for the effects on CIA are Cd79b, $A b c a 8 a$, and Map2k6. The congenic fragment also contains polymorphic genes that affect reproductive behavior and reproductive success. The Sox9 gene, known to influence sex reversal, is a candidate gene for the reproductive phenotype.
\end{abstract}

\section{Introduction}

Collagen-induced arthritis (CIA) is a commonly used animal model for rheumatoid arthritis (RA). Although CIA shares several features with RA, there are some obvious differences between the mouse model and the human disease [1-3]. One such dissimilarity is the reversed sex susceptibility. A female predominance is characteristic for RA [4], whereas the opposite situation commonly is the case in mice developing CIA. Because of the male predominance of CIA in most strains of mice, including B10.Q, most published CIA experiments have been performed on males.
We have previously performed a genetic linkage analysis on multiparous female mice from an N2 cross between NFR/N and B10.Q, with the aim of finding CIA loci that are linked to disease development in females [5]. We identified one novel significant CIA-associated locus on chromosome 11, which is now denoted $\mathrm{Cia} 40$. No other CIA loci/genes have previously been found in this region, but the central part of chromosome 11 is known to contain a number of inflammation loci, such as Eae22, Eae6b, Eae23, and Eae7 [6-8]. However, none of the experimental autoimmune encephalitis (EAE) loci is located close to the Cia4O linkage peak, indicating that other polymorphic genes might be of importance.

$\overline{\mathrm{CIA}}=$ collagen-induced arthritis; $\mathrm{Cll}=$ collagen type II; $\mathrm{EAE}=$ experimental autoimmune encephalitis; $\mathrm{MAP}=$ mitogen-activated protein; $\mathrm{QTL}=$ quantitative trait locus; $\mathrm{RA}=$ rheumatoid arthritis. 
Interestingly, in an additional quantitative trait locus (QTL) analysis with females of the same cross (N2 generation of NFR/N and B10.Q), we detected a highly significant OTL close to Cia40 on chromosome 11 linked to the trait 'pregnancy frequency' [9]. This locus is denoted Pregq2 and controls the frequency of successful pregnancies following successful copulation (successful coitus recorded by the detection of the 'vaginal plug'). In the initial QTL analysis, heterozygous mice carrying NFR/N genes at the Pregq2 locus suffered from an increased frequency of pregnancy failures [9]. We hypothesized that the Cia40/Pregq2 region of chromosome 11 may contain polymorphic genes that influence both CIA incidence and breeding success.

Although our original QTL analysis was performed on (aged) female mice with the hope of finding CIA loci with female predominance, there would still be a possibility that the Cia40 locus is of equal importance in both sexes. In the present paper, we present results indicating that Cia40 congenic females are more affected by CIA than males are. We also show that the Cia40/Pregq2 locus is linked to a disturbed reproductive behavior and reduced breeding performance in females.

\section{Materials and methods \\ Mice}

Inbred NFR/N mice were originally obtained from the National Institutes of Health (Bethesda, MD, USA) and the B10.Q mice were originally from the animal colony of Professor Jan Klein (Tübingen University, Tübingen Germany). (B10.Q × NFR/N) $\times$ B10.Q $N_{10}$ mice were bred in the animal house of the Department of Pathology of Lund University, Sweden. The animals were fed standard rodent chow and water in a photoperiod of light/dark 12:12. All mice used in the present study had clean health monitoring protocols according to the recommendations of the Federation of European Laboratory Animal Sciences Association. The ethical permission for reproduction and arthritis (M236-06,) was provided by the Swedish Board of Agriculture.

\section{The Cia40 congenic mice and the fragment}

To confirm the previously identified linkage on chromosome 11, we backcrossed the NFR/N strain to the (more) CIA-resistant strain, B10.Q. Mice heterozygous for the congenic region (a small fragment from the NFR/N strain on B10.Q background) were chosen for additional backcrossing for 10 generations (Figure 1). All of the mice were derived from the same set of parents. Subsequently, the congenic mice were intercrossed. Mice heterozygous for NFR/N markers between D11 Mit70 (93.8 Mb) and D11 mit214 (114.8 Mb) were intercrossed two times in order to produce the congenic line Cia40. All of the mice that were homozygote for Cia4O in the study had equal fragment size (Figure 1). However, the heterozygote animals differed slightly in fragment length among the individuals ( 1 to $2 \mathrm{Mb}$ ).
Figure 1

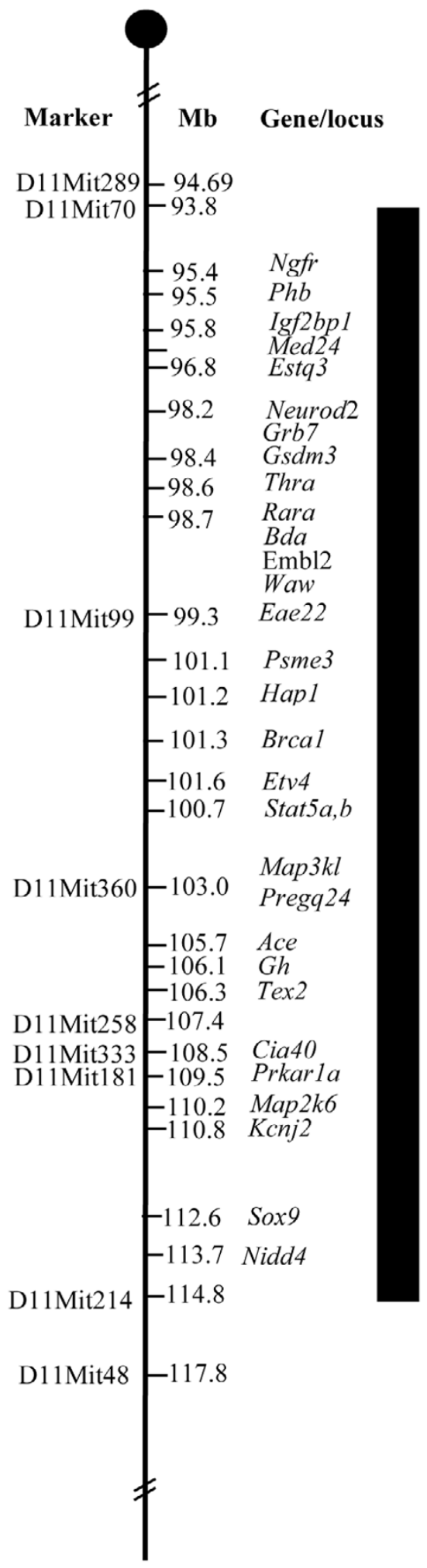

Overview of the Cia40/Pregq2 congenic fragment. The dark area indicates the genetic region from NFR/N in the congenic strain Cia40/ Pregq2. The markers are placed according to Mouse Ensemble built 36 [18]. 


\section{Genotyping}

Genomic DNA was isolated from the tip of the tail according to a previously described protocol [10]. Nine fluorescencelabeled polymorphic microsatellite markers (Interactiva, Biotechnologie $\mathrm{GmbH}$, Ulm, Germany) were used to cover the heterozygous fragment derived from the NFR/N as previously described [10] (Figure 1). The polymerase chain reaction products were analyzed on a MegaBACE ${ }^{\mathrm{TM}} 1000$ (GE Healthcare UK Ltd, Amersham Place, Little Chalfont, Buckinghamshire HP7 9NA, UK) according to the manufacturer's protocol. Data were analyzed with Genetic Profiler 1.1. (GE Healthcare UK Ltd, Amersham Place, Little Chalfont, Buckinghamshire HP7 9NA, UK).

\section{Induction and evaluation of collagen-induced arthritis}

To induce CIA, 8- to 12-week-old mice were immunized subcutaneously at the base of the tail with $100 \mu \mathrm{g}$ rat collagen type II (CII) emulsified in $0.1 \mathrm{M}$ acetic acid in complete Freund's adjuvant (Difco Laboratories, now part of Becton Dickinson and Company, Franklin Lakes, NJ, USA). After 30 days, a booster injection containing $50 \mu \mathrm{g} \mathrm{Cll}$ emulsified in $0.1 \mathrm{M}$ acetum in incomplete Freund's adjuvant (Becton Dickinson and Company) was given. The clinical scoring of arthritis was commenced 25 days after the first immunization. The scoring system is based on the number of inflamed joints, ranging from 1 to 15 for each affected paw. Each affected ankle/wrist was given a score of 5 , and each inflamed knuckle and toe was given 1 point. The scores of the four paws were added, yielding a maximum total score of 60 points for each mouse. The severity trait is the maximum score observed in each individual female. Mice that did not develop CIA were given a score of 0 for the traits of severity, onset, and incidence. The onset is the number of days calculated from the first immunization to the first clinical signs of arthritis excluding unaffected animals.

\section{Enzyme-linked immunosorbent assay}

The mice were sacrificed at day 90 and sera were collected. Anti-Cll antibody titers in sera were analyzed by a sandwich enzyme-linked immunosorbent assay technique [11]. In short, immunosorbent plates were coated with Cll $(10 \mu \mathrm{g} / \mathrm{mL})$ overnight at $4^{\circ} \mathrm{C}$. Bovine serum albumin (Sigma-Aldrich, St. Louis, MO, USA) was used for blocking, and thereafter different dilutions of control sera (purified mouse anti-collagen type II anti- bodies), test sera, and positive and negative controls were added. The presence of Cll-specific lgG was visualized by peroxidase-conjugated goat anti-mouse lgG.

\section{Statistical analysis}

Statistical comparison between the different experimental groups was performed by using the Mann-Whitney $U$ test.

\section{Results}

Increased incidence, onset, and severity of collageninduced arthritis in heterozygous Cia40 congenic female mice

Heterozygous and homozygous Cia40 congenic mice and corresponding littermate controls of both sexes were immunized with rat $\mathrm{Cll}$ and monitored three times a week for 90 days. Serum samples for anti-Cll antibody analysis were collected at the end point of the experiment. Results presented in Table 1 show that heterozygous Cia40 congenic mice suffer from an elevated incidence of the disease. This increase in incidence was particularly obvious and significant in the group of females $(P<0.05)$. Surprisingly, no significant differences in incidence were observed in homozygous Cia40 congenic females or males in comparison with the corresponding controls. The onset of the disease was significantly quicker in heterozygous females in comparison with wildtype B10.Q and homozygous congenic littermates. There were no significant differences in onset between the different groups of males. The severity of the disease was elevated in heterozygous Cia40 congenic mice of both sexes, as shown in Figures $2 \mathrm{a}$ and $2 \mathrm{~b}$. Homozygous mice showed a minor increase in severity in comparison with wildtype B10.Q littermates, but this difference was not significant. The heterozygous congenic males showed a higher severity in the beginning of the disease, whereas heterozygous females showed higher severity in the latter part of the disease. The heterozygous congenic females developed a more severe arthritis than the heterozygous congenic male mice. The heterozygous congenic females also showed a significantly shorter onset $(P<0.05)$ of CIA than corresponding controls and all other groups (Table 2).

\section{Heterozygous Cia40/Pregq2 congenic mice show increased anti-collagen type II antibody levels}

Anti-Cll antibody titers in serum were analyzed at the end of

Table 1

Incidence of collagen-induced arthritis in Cia40 congenic male and female mice

\begin{tabular}{lcccc}
\hline & Number & Incidence & Homozygous Cia40 \\
\hline Total & & Wildtype B10.Q & Heterozygous Cia40 & $12 / 36(33 \%)$ \\
Females & 116 & $12 / 48(25 \%)$ & $24 / 47(51 \%)$ & $5 / 15(33 \%)$ \\
Males & 54 & $4 / 24(17 \%)$ & $12 / 15(80 \%)$ a & $7 / 21(33 \%)$ \\
\hline
\end{tabular}

aSignificantly higher incidence in heterozygous congenic females compared with wildtype littermates $(P<0.05)$. 
Table 2

Onset of arthritis in Cia40 congenic male and female mice

\begin{tabular}{lcccc}
\hline & Number & Onset ${ }^{\text {(range })}$ \\
\hline Total & & Wildtype B10.Q & Heterozygous Cia40 & Homozygous Cia40 \\
Females & 116 & $55(32,82)$ & $50(29,78)$ & $45(38,84)$ \\
Males & 54 & $53(32,70)$ & $38(29,59)^{\mathrm{b}}$ & $42(38,72)$ \\
\end{tabular}

aDay of onset. Median values for onset calculated on all arthritic mice in the group on day 90 . Figures in parenthesis indicate minimum and maximum values for onset. bSignificantly shorter onset in heterozygous congenic females compared with wildtype littermates $(P<0.05)$.

Figure 2

(a)

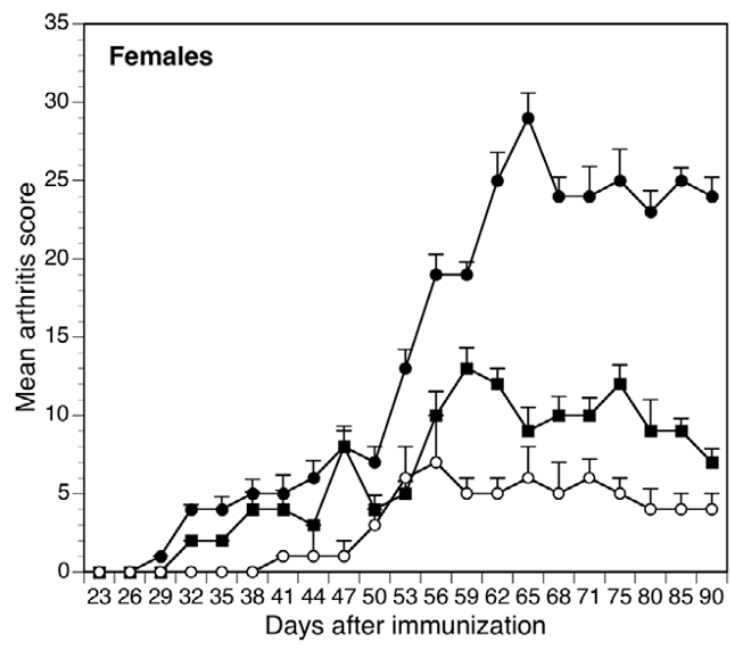

$\rightarrow$ Homozygous for Cia40

$\rightarrow$ Heterozygous for Cia40

-o- Homozygous for B10.Q

(b)

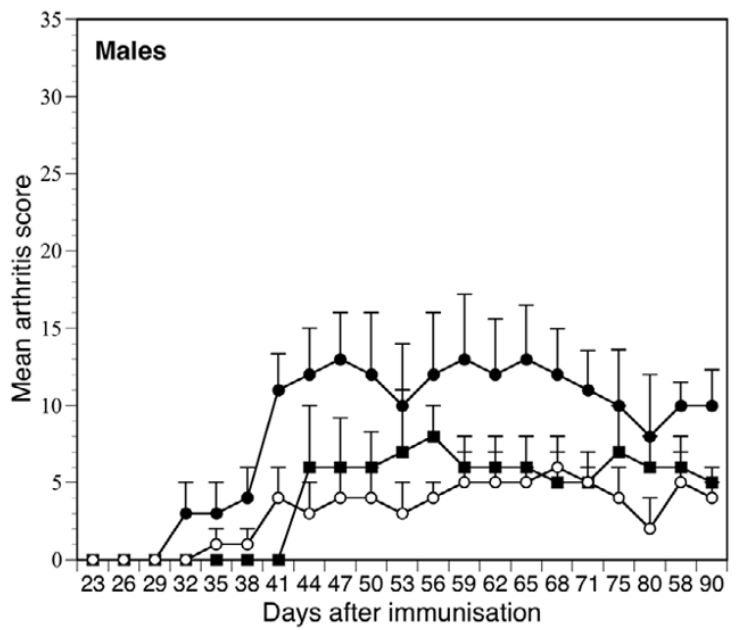

$\rightarrow-$ Homozygous for Cia40

$\rightarrow$ Heterozygous for Cia40

$\multimap$ Homozygous for B10.Q

Severity of collagen-induced arthritis in Cia40 congenic male and female mice. (a) Mean (standard error, SE) arthritic scores in homozygous Cia40 congenic females, heterozygous Cia40 congenic females, and wildtype littermate females. Only mice that developed arthritis have been included. Heterozygous congenic females show higher severity than wildtype B10.Q and congenic homozygous females $(P<0.05)$. (b) Mean (SE) arthritic scores in homozygous Cia4O congenic males, heterozygous Cia 40 congenic males, and wildtype littermate males. Only mice that developed arthritis have been included. Heterozygous congenic males show significantly higher severity than wildtype B10.Q littermates $(P<0.05)$. 
Table 3

Anti-collagen type II titers in Cia40 congenic male and female mice

\begin{tabular}{lcccc}
\hline & Number & Anti-collagen type Il titers at day 90, mg/mL & Homozygous Cia40 \\
\hline Total & & Wildtype B10.Q & Heterozygous Cia40 & $0.86 \pm 0.25$ \\
Females & 161 & $0.68 \pm 0.24$ & $1.29 \pm 0.31$ & $0.96 \pm 0.24$ \\
Males & 54 & $0.71 \pm 0.32$ & $1.57 \pm 0.34^{\mathrm{a}}$ & $0.70 \pm 0.17$ \\
\hline
\end{tabular}

aSignificantly higher antibody titer in heterozygous congenic females compared with wildtype littermates $(P<0.05)$. Values are presented as mean \pm standard error.

the experiment (Table 3). The results showed that heterozygous Cia40 congenic females develop significantly higher anti-Cll antibody titers than wildtype and homozygous congenic mice $(P<0.05)$ of the same sex. No significant differences in anti-Cll titers were observed between the different groups of males. This shows that the antibody titers follow the disease phenotype in the congenic mice.

\section{Reduced breeding performance and disturbed breeding behavior in Cia40/Pregq2 congenic mice}

The Cia40/Pregq2 congenic mice were difficult to breed and congenic mice of both sexes showed disturbed breeding behavior. Congenic females showed a reduced frequency of successful pregnancies, and pups were frequently killed and eaten shortly after delivery. Figure $3 a$ shows that the mean litter size (surviving pups) of Cia40 congenic females crossed with $\mathrm{B} 10 . \mathrm{Q}$ males is significantly reduced $(P=0.041)$ compared with the litter size of wildtype littermate females crossed with B10.Q males. Figure $3 b$ shows the frequency of litters containing dead pups (the exact numbers were normally not possible to count) in breeding cages containing Cia40/ Pregq2 congenic female mice and breeding cages containing only wildtype littermate females. The frequency of litters containing dead pups was dramatically higher in breeding cages containing Cia40/Pregq2 congenic females compared with those containing wildtype females $(P=0.0069)$. These data show that the majority of the litters that were born by the congenic females contained nonsurviving pups. The high neonatal mortality among the pups from the congenic females appeared to be due to behavioral disturbance characterized by maternal ignorance and a tendency toward attacking and eating their own pups.

\section{Discussion}

The results of the present study indicate that one or more polymorphic genes in the congenic Cia40/Pregq2 fragment

Table 4

Summary of possible candidate genes on chromosome 11 for Cia40/Pregq2

\begin{tabular}{|c|c|c|c|}
\hline Gene & Position, mb & Description & $\begin{array}{l}\text { Reproductive or inflammatory phenotypes of } \\
\text { mutation }\end{array}$ \\
\hline Ngfr & $95.430132-95.449049$ & Nerve growth factor receptor & Perinatal lethality \\
\hline Phb & $95.5528271-95.542087$ & Prohibitin & Lethality before weaning \\
\hline $\operatorname{lgf2bp} 1$ & $95.818477-95.867254$ & Insulin-like growth factor 2 & Fetal growth \\
\hline Med24 & $96.565905-98.590749$ & Mediator complex subunit 24 & Pups die prior to birth \\
\hline Gsdm3 & $98.490658-98.499540$ & Gasdermin & Abnormal loss of skin and hair \\
\hline Etv4 & $101.631061-101.646685$ & $\begin{array}{l}\text { Ets variant gene } 4 \text { (E1A enhancer-binding protein, } \\
\text { E1AF) }\end{array}$ & Mammary gland abnormality, male infertility \\
\hline$C d 79 b^{a}$ & $106.172655-106.176076$ & CD79B antigen & Hematopoietic, immune \\
\hline Prkar1a & $109.510719-109.530970$ & $\begin{array}{l}\text { Protein kinase, cAMP-dependent regulatory, type I, } \\
\text { alpha }\end{array}$ & Embryonic lethality \\
\hline Abca8a $a^{a}$ & $109.886948-109.957292$ & $\begin{array}{l}\text { ATP-binding cassette, sub-family A (ABC1), } \\
\text { member } 8 \mathrm{a}\end{array}$ & Not known \\
\hline Map2k6a & $110.260436-110.386836$ & Mitogen-activated protein kinase kinase 6 & Abnormal immune system \\
\hline Sox9 & $112.643538-112.649074$ & SRY-box containing gene 9 & Perinatal lethality, cartilage formation, sex reversal \\
\hline
\end{tabular}

aPolymorfism between inbreed strains of NMRI and C57BL/10 according to gscan, Wellcome Trust Centre for Human Genetics [13]. Mb for the genes/markers are according to Mouse Ensemble built 36 [18]. 


\section{Figure 3}

(a)

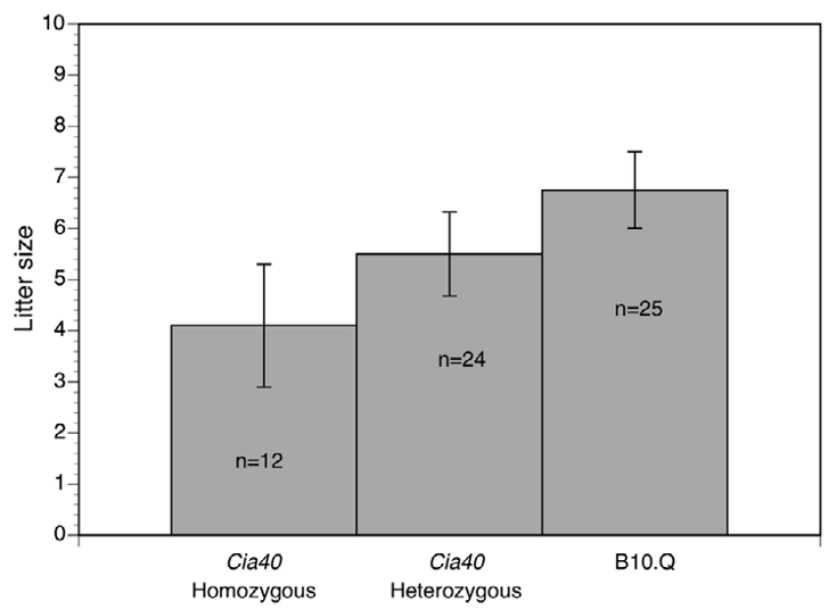

(b)

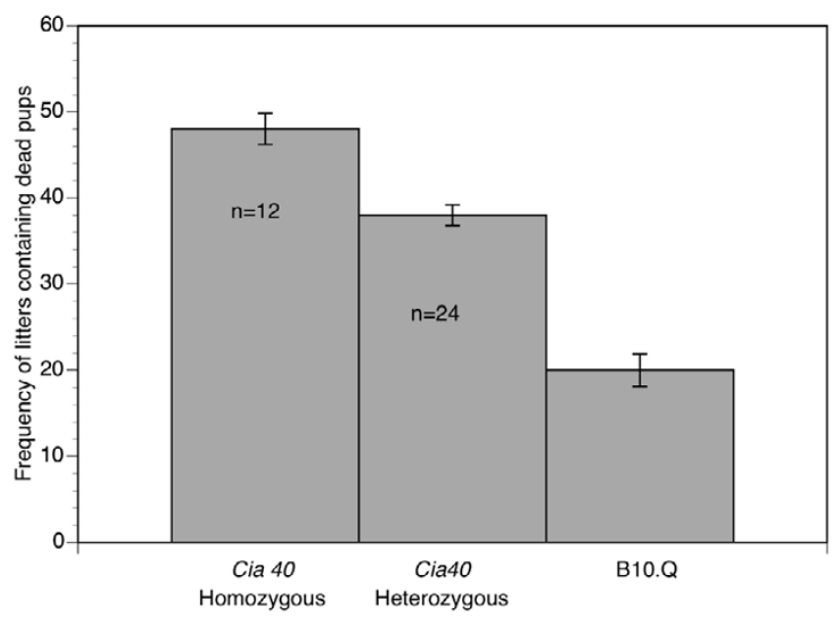

The mean litter size and the frequency of litters containing dead pups in Cia 40 congenic females and B10.Q wildtype littermates. (a) The mean litter size (standard error, SE) in homozygous Cia4O congenic females, heterozygous $\mathrm{Cia} 40$ congenic females, and wildtype B10.Q littermates ( $n=$ number of pregnancies). The difference between homozygous Cia40 congenic mice and wildtype B10.Q littermates was significant $(P<0.05)$. (b) The mean (SE) frequency (percentage) of litters containing dead pups in homozygous $\mathrm{Cia} 4 \mathrm{O}$ congenic mice, heterozygous Cia4O congenic mice, and wildtype B10.Q littermates ( $n=$ number of pregnancies). The difference between $\mathrm{Cia} 4 \mathrm{O}$ homozygous congenics and B10.Q wildtype littermates was significant $(P<0.007)$.

affect severity, onset, and incidence of $\mathrm{CIA}$ as well as the reproductive performance of $\mathrm{B} 10 . \mathrm{Q}$ mice. Interestingly, the increased incidence and severity are pronounced traits in heterozygous mice only, and the influence of the congenic fragment is particularly obvious in the heterozygous females, which actually show a much higher incidence than the males. This is striking since females of the strain B10.Q normally show a very low incidence of arthritis (around 15\%). The female predominance in incidence of CIA makes polymorphic genes in the congenic fragment particularly interesting since female predominance is characteristic for RA in humans.

None of the genes close to the calculated position of the Cia40/Pregq2 locus is known to be involved in the regulation of inflammation (Table 1). For this reason, we believe that polymorphic or mutated regulatory genes, which in turn affect the activity of several enzymes, could be particularly interesting candidate genes. One such candidate gene is mitogen-activated protein (MAP) kinase, Map2k6, which has been reported to affect the function of the immune system. For instance, Ehlting and colleagues [12] recently reported that the regulation of a suppressor of cytokine signalling $3^{\prime}$ (SOCS3) mRNA stability by tumor necrosis factor-alpha involves the activation of the MAP kinase cascade. Table 4 shows possible gene candidates, based on single-nucleotide polymorphism data in this particular fragment on chromosome 11 in between inbreed strains of NMRI and C57BL/10 mice from the Wellcome Trust database (gscan) [13].

We have previously speculated that the same gene(s) might affect both arthritis incidence and pregnancy failure [5]. This assumption is supported by the fact that the incidence of autoimmune CIA is elevated in females but not in males and that the elevated severity is particularly obvious in females. A modified gene that increases the risk of developing autoimmune inflammation in females can also be expected to interfere negatively with pregnancy success. Some types of early pregnancy failures could actually be caused by increased autoimmune reactivity. Again, it is possible that the MAP kinase is involved in the success of implantation. This assumption is strengthened by a recent observation that the MAP kinase cascade indeed affects preimplanted embryos [14]. Still, it might be more likely that different mechanisms and genes are involved in the regulation of arthritic inflammation and the regulation of pregnancy success. If true, this would make it possible to separate Cia40 gene(s) from the breedingsuppressing Pregq2 gene(s), which would be of great advantage for the future characterization of the part of the Cia40 gene(s) that influence the outcome of arthritis.

The observation that the heterozygous Cia40 congenic mice show a quicker onset, and in the case of males, also develop a more severe disease, raises questions about the molecular mechanisms controlling arthritis. A polymorphism leading to an amino acid substitution in one allele could have strong effects on the function of a di- or multimeric protein and polymorphisms in noncoding regulatory regions could result in skewed transcription and altered protein levels. The observed phenotypic effects due to heterozygous alleles might be helpful in the identification of candidate genes. The heterozygous effect has previously been reported in a study of CIA development, in which mice with heterozygous alleles in a congenic fragment on mouse chromosome 15 were much more affected by the disease than homozygous littermates were [15]. 
We have found only a limited number of genes in the vicinity of the Cia40 and Pregq2 peaks, which show polymorphism between B10 and NMRI. In addition to Mapk6, we have focused some attention on the Abca8a gene and $C D 79 b$ gene. The role of the $A b c a 8 a$ gene in the context of reproduction and immunity is largely unknown, whereas the $C D 79 b$ gene is of importance primarily in the context of B-cell development [16]. At present, it is not possible to speculate about the possible influence of these two genes for the phenotypes observed, but the function of these genes does not make them our main candidate genes.

The interesting reversal of sex susceptibility to arthritis and the observations that congenic males show impaired development of genital organs and that females are more aggressive and less caring mothers have made us pay attention to the Sox 9 gene. The Sox9 gene has been reported to cause sex reversal [17], which is a highly relevant phenotype in the context of the Cia40/Pregq2 congenic mice. The possible presence of a Sox9 polymorphism/mutation on chromosome 11 in our congenic mice is under investigation.

\section{Conclusion}

The present results show that the Cia40 locus on chromosome 11 contains one or more polymorphic genes that particularly influence incidence and severity of CIA in female mice. These effects are significant in congenic B10.Q female mice carrying heterozygous $\mathrm{Cia} 40$ fragments of NFR/N origin. Congenic mice carrying heterozygous fragments also show quicker onset of the disease. The major polymorphic candidate genes in the congenic fragment are Cd79b, Abca8a, and Map2k6. The NFR/N fragment present in the congenic mice also contains a locus denoted Pregq2, which causes a change in reproductive behavior and reduces pregnancy success. This effect is significant in congenic B10.Q females carrying a homozygous NFR/N fragment. The Sox 9 gene, known to influence sex reversal, is a candidate gene for the reproductive phenotype.

\section{Competing interests}

The authors declare that they have no competing interests.

\section{Authors' contributions}

ML was responsible for genotyping, phenotyping, and analysis and helped to interpret the data and write the manuscript. RM, $\AA \mathrm{A}$, and $\mathrm{RH}$ helped to interpret the data and write the manuscript. All authors read and approved the final manuscript.

\section{Acknowledgements}

This study was supported by the Österlund's Fund, Kock's Fund, Crafoord's Fund, Gustav V 80-Year Foundation, the Royal Physiographic Society in Lund, and the Lars Hierta Memorial Foundation.

\section{References}

1. Wooley PH: Collagen-induced arthritis in the mouse. Methods Enzymol 1988, 162:361-373.
2. Holmdahl R, Jansson L, Andersson M, Larsson E: Immunogenetics of type II collagen autoimmunity and susceptibility to collagen arthritis. Immunology 1988, 65:305-310.

3. Kannan K, Ortmann RA, Kimpel D: Animal models of rheumatoid arthritis and their relevance to human disease. Pathophysio/ogy 2005, 12:167-181.

4. Da Silva JA, Hall GM: The effects of gender and sex hormones on outcome in rheumatoid arthritis. Bailliers Clin Rheumatol 1992, 6:196-219.

5. Liljander M, Sällström M-A, Andersson S, Andersson Å, Holmdahl $\mathrm{R}$, Mattsson R: Identification of collagen-induced arthritis loci in aged multiparous female mice. Arthritis Res Ther 2006, 8:R45.

6. Karlsson J, Zhao X, Lonskaya I, Neptin M, Holmdahl R, Andersson $\AA$ : Novel quantitative trait loci controlling development of experimental autoimmune encephalomyelitis and proportion of lymphocyte subpopulations. J Immunol 2003, 170:1019-1026.

7. Butterfield RJ, Blankenhorn EP, Roper RJ, Zachary JF, Doerge RW Teuscher C: Identification of genetic loci controlling the characteristics and severity of brain and spinal cord lesions in experimental allergic encephalomyelitis. Am J Pathol 2000 157:637-645.

8. Adarichev VA, Nesterovitch $A B$, Bardos $T$, Biesczat $D$, Chandrasekaran R, Vermes C, Mikecz K, Finnegan A, Glant TT: Sex effect on clinical and immunologic quantitative trait loci in a murine model of rheumatoid arthritis. Arthritis Rheum 2003, 48:1708-1720.

9. Liljander M, Sällström M-A, Andersson S, Andersson A, Holmdahl $\mathrm{R}$, Mattsson R: Identification of genetic regions of importance for reproductive performance in female mice. Genetics 2006, 173:901-909.

10. Laird PW, Zijderveld A, Linders K, Rudnicki MA, Jaenisch R, Berns A: Simplified mammalian DNA isolation procedure. Nucleic Acids Res 1991, 19:4293.

11. Engvall E: Enzyme immunoassay ELISA and EMIT. Enzymology 1980, 70:419-439.

12. Ehlting C, Lai WS, Schaper F, Brenndörfer ED, Matthes RJ, Heinrich PC, Ludwig S, Blackshear PJ, Gaestel M, Häussinger D, Bode JG: Regulation of suppressor of cytokine signaling 3 (SOCS3) mRNA stability by TNF-alpha involves activation of the MKK6/ p38MAPK/MK2 cascade. J Immuno/ 2007, 178:2813-2826.

13. Mouse SNP Selector, Wellcome Trust Centre for Human Genetics: Bioinformatics and Statistical Genetics [http:// gscan.well.ox.ac.uk/gs/strains.cgi]

14. Fong $\mathrm{B}$, Watson $\mathrm{PH}$, Watson AJ: Mouse preimplantation embryo responses to culture medium osmolarity include increased expression of CCM2 and p38 MAPK activation. BMC Dev Biol 2007, 7:2.

15. Karlsson J, Johannesson $M$, Lindvall $T$, Wernhoff $P$, Holmdahl $R$, Andersson A: Genetic interactions in Eae2 control collageninduced arthritis and the $\mathrm{CD}^{+} / \mathrm{CD}^{+} \mathrm{T}$ cell ratio. J Immunol 2005, 174:533-541.

16. Dobbs AK, Yang T, Farmer D, Kager L, Parolini O, Conley ME: Cutting edge: a hypomorphic mutation in Igbeta (CD79b) in a patient with immunodeficiency and leaky defect in B cell development. J Immunol 2007, 179:2055-2059.

17. Manuylov NL, Fujiwara Y, Adameyko II, Poulat F, Tevosian SG: The regulation of Sox9 gene expression by the GATA4/FOG2 transcriptional complex in dominant $X X$ sex reversal mouse models. Dev Biol 2007, 307:356-367.

18. Mouse (Mus musculus), Ensembl Genome Browser [http:// www.ensembl.org/Mus musculus/index.html] 